\title{
Conformal Laplace superintegrable systems in 2D: polynomial invariant subspaces
}

\author{
M.A. Escobar-Ruiz \\ Instituto de Ciencias Nucleares, UNAM \\ Apartado Postal 70-543, 04510 Mexico D.F. MEXICO \\ and School of Mathematics, University of Minnesota \\ mauricio.escobar@nucleares.unam.mx \\ and Willard Miller, Jr. \\ School of Mathematics, University of Minnesota, \\ Minneapolis, Minnesota, 55455, U.S.A. \\ miller@ima.umn.edu
}

September 23, 2021

\begin{abstract}
2nd-order conformal superintegrable systems in $n$ dimensions are Laplace equations on a manifold with an added scalar potential and $2 n-1$ independent 2 nd order conformal symmetry operators. They encode all the information about Helmholtz (eigenvalue) superintegrable systems in an efficient manner: there is a 1-1 correspondence between Laplace superintegrable systems and Stäckel equivalence classes of Helmholtz superintegrable systems. In this paper we focus on superintegrable systems in two dimensions, $n=2$, where there are 44 Helmholtz systems, corresponding to 12 Laplace systems. For each Laplace equation we determine the possible 2 -variate polynomial subspaces that are invariant under the action of the Laplace operator, thus leading to families of polynomial eigenfunctions. We also study the behavior of the polynomial invariant subspaces under a Stäckel transform. The principal new results are the details of the polynomial variables and the conditions on parameters of the potential corresponding to polynomial solutions. The hidden $g l_{3}$-algebraic structure is exhibited for the exact and quasi-exact systems. For physically meaningful solutions, the orthogonality properties and normalizability of the polynomials are presented as well. Finally, for all Helmholtz superintegrable solvable systems we give a unified construction of $1 \mathrm{D}$ and $2 \mathrm{D}$ quasi-exactly solvable potentials possessing polynomial solutions, and a construction of new 2D PT-symmetric potentials is established.
\end{abstract}




\section{Introduction}

This paper is part of a series [1,2] whose purpose is to systematize and unify the study of 2 nd order Helmholtz (i.e. Schrödinger eigenvalue) superintegrable systems in $2 \mathrm{D}$ and $3 \mathrm{D}$ by transforming these systems to conformally superintegrable Laplace equations with a scalar potential and applying ideas originally due to Bôcher, [3]. It is well known [4, 5, 6, 7, 8, 9, 10] that any second order conformal Laplace superintegrable system admitting a non-constant potential $V(\mathbf{x})$ can be Stäckel transformed to a Helmholtz superintegrable system, and this operation is invertible. Moreover, each family of Stäckel-equivalent Helmholtz superintegrable systems on a variety of manifolds corresponds to a single conformally superintegrable system on flat space. The different Helmholtz systems in an equivalence class share some important properties. For example, their structure algebras are isomorphic, modulo a permutation of parameters. Furthermore, by taking a gauge transformation, most of these Laplace equations can be transformed into an eigenvalue problem with a Hamiltonian operator that leaves invariant a polynomial vector space. This polynomial vector space is the same for all Hamiltonians in a Stäckel equivalence class and it can most conveniently be studied via Laplace equations. Except for a few exceptional special cases, these Helmholtz superintegrable systems are multiseparable, with the various separable solutions characterized as eigenfunctions of a 2nd order symmetry operator for the $2 \mathrm{D}$ systems. Typically this operator also leaves the polynomial vector space invariant, and the eigenfunctions are polynomial special functions. The Hamiltonian and its 2 nd order symmetries are formally self-adjoint so this construction leads to families of orthogonal polynomials. The usual hypergeometric orthogonal polynomials such as Jacobi, Laguerre and Hermite arise in this way, but also non-hypergeometric polynomials such as Heun and spheroidal appear. This is one important way that special functions and orthogonal polynomials are related to superintegrable systems. These special functions all satisfy differential equations. A second important way special functions are related to such systems is due to multiseparability. The separated eigenfunctions characterized by one symmetry operator can be expanded in the eigenbasis of another symmetry operator. The expansion coefficients are themselves special functions with orthogonality properties, and they may satisfy difference as well as differential equations. For example, Wilson and Racah polynomials arise in this way, e.g. [11, 12]. The contraction scheme relating superintegrable systems leads to limit relations for orthogonal polynomials and special functions, including the Askey Scheme for hypergeometric orthogonal polynomials, [13, 14].

Here we consider all 2D 2nd order superintegrable systems and determine when systems are exactly solvable or quasi-exactly solvable, i.e., we find the gauge factor and the variables for which the gauge transformed operator possesses polynomial invariant subspaces. We also pay attention to the 
issue of when the 1D separation equations which arise from these systems are QES and the polynomial special functions that occur. Finally, since all of these systems have classical mechanical counterparts we discuss the relations between the trajectories and their time dependence for classical Helmholtz superintegrable systems that belong to the same equivalence class.

\subsection{Conformally superintegrable Laplace systems}

We consider Laplace systems of the form

$$
H \Psi(\mathbf{x}) \equiv\left(\Delta_{2}+V(\mathbf{x})\right) \Psi(\mathbf{x})=0 .
$$

Here $\Delta_{2}$ is the Laplace-Beltrami operator on a real or complex $2 D$ Riemannian or pseudo-Riemannian manifold and $V$ is a non-zero scalar potential. All variables can be complex, except when we impose constraints such as square integrability. A conformal symmetry of equation (1) is a partial differential operator $L$ in the variables $\mathbf{x}=\left(x_{1}, x_{2}\right)$ such that $[L, H] \equiv L H-H L=R_{L} H$ for some differential operator $R_{L}$. A conformal symmetry maps any solution $\Psi$ of (1) to another solution. Two conformal symmetries $L, L^{\prime}$ are identified if $L=L^{\prime}+R H$ for some differential operator $R$, since they agree on the solution space of (1). (For brevity we will say that $L=L^{\prime}, \bmod (H)$ and that $L$ is a symmetry if $[L, H]=0, \bmod (H)$.) The system is conformally superintegrable if there exist three algebraically independent conformal symmetries, $L_{1}, L_{2}, L_{3}$ with $L_{3}=H$. It is second order conformally superintegrable if each $L_{2}$ can be chosen to be a 2nd order differential operator, and $L_{1}$ of at most 2 nd order. (If the system admits symmetries such that $L_{1}, L_{2}$ can be chosen as 1 st order, we say it is 1 st order conformally superintegrable). Recall that a Helmholtz eigenvalue system $\hat{H} \Psi=E \Psi$ with $\hat{H}=\Delta+\hat{V}$ is superintegrable if there exist three algebraically independent true symmetries, $\hat{L}_{1}, \hat{L}_{2}, \hat{L}_{3}$ with $\hat{L}_{3}=\hat{H}$, i.e., $\left[\hat{L}_{j}, \hat{H}\right]=0$.

Every 2D Riemannian manifold is conformally flat, so we can always find a Cartesian-like coordinate system with coordinates $(x, y) \equiv\left(x_{1}, x_{2}\right)$ such that a Helmholtz system takes the form

$$
\hat{H} \Psi=\frac{1}{\lambda(x, y)}\left(\partial_{x}^{2}+\partial_{y}^{2}+\hat{V}(x, y)\right) \Psi=E \Psi .
$$

We can rewrite this as a flat space Laplace system $H \Psi=0$ where $H=$ $\partial_{x}^{2}+\partial_{y}^{2}+V$ with $V=\hat{V}-E \lambda$. It is easy to show that this Laplace system is conformally superintegrable if and only if the Helmholtz system is superintegrable. Thus any Helmholtz superintegrable system on any manifold corresponds to a flat space Laplace conformally superintegrable system. Moreover, given a flat space Laplace superintegrable system with metric $d s^{2}=d x^{2}+d y^{2}$, measure $d x d y$ and potential of the form $V=V_{0}+\alpha U$ for 


\begin{tabular}{|c||c|}
\hline System & Non-degenerate potentials \\
& $V(x, y)$ \\
\hline \hline$[1,1,1,1]$ & $\frac{a_{1}}{x^{2}}+\frac{a_{2}}{y^{2}}+\frac{4 a_{3}}{\left(x^{2}+y^{2}-1\right)^{2}}-\frac{4 a_{4}}{\left(x^{2}+y^{2}+1\right)^{2}}$ \\
{$[2,1,1]$} & $\frac{a_{1}}{x^{2}}+\frac{a_{2}}{y^{2}}-a_{3}\left(x^{2}+y^{2}\right)+a_{4}$ \\
{$[2,2]$} & $\frac{a_{1}}{(x+i y)^{2}}+\frac{a_{2}(x-i y)}{(x+i y)^{3}}+a_{3}-a_{4}\left(x^{2}+y^{2}\right)$ \\
{$[3,1]$} & $a_{1}-a_{2} x+a_{3}\left(4 x^{2}+y^{2}\right)+\frac{a_{4}}{y^{2}}$ \\
{$[4]$} & $a_{1}-a_{2}(x+i y)+a_{3}\left(3(x+i y)^{2}+2(x-i y)\right)-a_{4}\left(4\left(x^{2}+y^{2}\right)+2(x+i y)^{3}\right)$ \\
{$[0]$} & $a_{1}-\left(a_{2} x+a_{3} y\right)+a_{4}\left(x^{2}+y^{2}\right)$ \\
$(1)$ & $\frac{a_{1}}{(x+i y)^{2}}+a_{2}-\frac{a_{3}}{(x+i y)^{3}}+\frac{a_{4}}{(x+i y)^{4}}$ \\
$(2)$ & $a_{1}+a_{2}(x+i y)+a_{3}(x+i y)^{2}+a_{4}(x+i y)^{3}$ \\
\hline
\end{tabular}

Table 1: Four-parameter potentials. Each of the Helmholtz nondegenerate superintegrable (i.e. 3-parameter) eigenvalue systems is Stäckel equivalent to exactly one of these systems. Thus, with one caveat mentioned in [1], there are exactly 8 equivalence classes of Helmholtz systems.

functions $V_{0}, U$ and parameter $\alpha$, then its Conformal Stäckel Transform

$$
C S T: \quad \tilde{H} \Psi=U^{-1}\left(\partial_{x}^{2}+\partial_{y}^{2}+V_{0}\right) \Psi=-\alpha \Psi
$$

is a Helmholtz superintegrable system with metric $d s^{2}=U\left(d x^{2}+d y^{2}\right)$, measure $U d x d y$. There is an analogous definition of Stäckel transforms that take one Helmholtz superintegrable system to another, [5]. We see from this that each equivalence class of Helmholtz superintegrable systems corresponds to a single conformally superintegrable flat space Laplace system.

In papers [1,2] it is shown that the 44 families of Helmholtz superintegrable systems correspond to exactly 14 Laplace systems. They are of two types: the 8 systems with non-degenerate potentials (4 parameter, 3 parameter for the Helmholtz systems), Table 1, and the 6 systems with degenerate potentials ( 2 parameter, 1 parameter for the Helmholtz systems), Table 2. There are no other possibilities. The degenerate potentials can all be obtained as parameter restrictions of nondegenerate ones, but they have additional symmetry not inherited from the restriction. All of the nondegenerate Laplace systems can be obtained as Bôcher contractions of system $[1,1,1,1]$, [2]. All of the degenerate Laplace systems can be obtained as Bôcher contractions of system $A$. 


\begin{tabular}{|c||c|}
\hline System & $\begin{array}{c}\text { Degenerate potentials } \\
V(x, y)\end{array}$ \\
\hline \hline$A$ & $\frac{4 a_{3}}{\left(x^{2}+y^{2}-1\right)^{2}}-\frac{4 a_{4}}{\left(x^{2}+y^{2}+1\right)^{2}}$ \\
$B$ & $\frac{a_{1}}{x^{2}}+a_{4}$ \\
$C$ & $a_{3}-a_{4}\left(x^{2}+y^{2}\right)$ \\
$D$ & $a_{1}-a_{2} x$ \\
$E$ & $\frac{a_{1}}{(x+i y)^{2}}+a_{3}$ \\
$F$ & $a_{1}-a_{2}(x+i y)$ \\
\hline
\end{tabular}

Table 2: Two-parameter degenerate potentials.

\section{Relations between conformal Stäckel transforms and polynomial solutions of Laplace systems}

For an eigenvalue equation $\mathcal{H} \psi(\mathbf{x})=a \psi(\mathbf{x})$ with spectral parameter $a$ :

- The operator $\mathcal{H}$ is said to be exactly-solvable, (ES) if there exists an infinite flag of subspaces $\mathcal{P}_{N}, N=1,2,3, \ldots$, such that $n_{N}=\operatorname{dim} \mathcal{P}_{N} \rightarrow$ $\infty$ as $N \rightarrow \infty$ and $\mathcal{H} \mathcal{P}_{N} \subseteq \mathcal{P}_{N} \subseteq \mathcal{P}_{N+1}$ for any $N$. In this case, for each subspace $\mathcal{P}_{N}$ the $n_{N}$ eigenvalues and eigenfunctions of $\mathcal{H}$ can be obtained by pure algebraic means.

- The operator $\mathcal{H}$ is called quasi-exactly solvable, (QES) if there exist a single subspace $\mathcal{P}_{k}$ of dimension $n_{k}>0$ such that $\mathcal{H} \mathcal{P}_{k} \subseteq \mathcal{P}_{k}$. In this case, again we can find $n_{k}$ eigenvalues and eigenfunctions of $\mathcal{H}$ by algebraic means, but we have no information about the remaining eigenvalues and eigenfunctions.

See $[15,16,17,18,19]$.

We will consider the 14 Laplace 2D systems $H \Psi=\left(\partial_{x}^{2}+\partial_{y}^{2}+V\right) \Psi=0$, 8 with non-degenerate potential $V=V\left(\mathbf{x} ; a_{1}, a_{2}, a_{3}, a_{4}\right)$, which depend linearly on four parameters, and 6 systems with degenerate potential, depending linearly on two parameters. We will show that, after an appropriate gauge transformation, most of these Laplace systems lead to an eigenvalue problem which is exactly solvable or quasi-exactly solvable, as are all Helmholtz systems obtained from them by conformal Stäckel transforms. 
In particular, we will show that most of the Laplace systems mentioned above possess a hidden $g l_{3}$ algebra realized by the generators [19]

$$
\begin{aligned}
& \mathcal{J}_{i}^{-}=\partial_{w_{i}}, \quad i=1,2, \\
& \mathcal{J}_{i j}^{0}=w_{i} \partial_{w_{j}}, \quad i, j=1,2, \\
& \mathcal{J}^{0}(N)=w_{1} \partial_{w_{1}}+w_{2} \partial_{w_{2}}-N, \\
& \mathcal{J}_{i}^{+}(N)=w_{i} \mathcal{J}^{0}(N)=w_{i}\left(w_{1} \partial_{w_{1}}+w_{2} \partial_{w_{2}}-N\right) .
\end{aligned}
$$

The parameter $N$ in (2) can be any real number. However, if $N$ is a nonnegative integer, the representation (2) of the $g_{3}$ algebra becomes the finitedimensional representation acting on the space of polynomials

$$
\mathcal{P}_{N}^{(2)}=\left\langle w_{1}^{p_{1}} w_{2}^{p_{2}} \mid 0 \leq p_{1}+p_{2} \leq N\right\rangle,
$$

which form the flag $\mathcal{P}_{0}^{(2)} \subset \mathcal{P}_{1}^{(2)} \subset \mathcal{P}_{2}^{(2)} \ldots \subset \mathcal{P}_{N}^{(2)} \subset \ldots \mathcal{P}$. A quadratic function in the generators (2) maps the polynomial space (3) into itself.

For exactly and quasi-exactly solvable systems we will determine the following three elements

1. The polynomial variables $w_{j}=w_{j}(\mathbf{x}), j=1,2$. They are the variables in which the (1) Laplace system admits invariant domains where $\Psi$ takes the form of a polynomial $P$ (of total order $N$ ) times a common (gauge) factor $\Psi_{0}$,

$$
\Psi=\Psi_{0}\left(w_{1}, w_{2}\right) P_{N}\left(w_{1}, w_{2}\right) .
$$

For the polynomial systems we will find that, except in $[1,1,1,1]$ and $A$ listed in Tables 1 and 2 respectively, factorization of variables occurs: $\Psi_{0}\left(w_{1}, w_{2}\right)=\psi_{1}\left(w_{1}\right) \psi_{2}\left(w_{2}\right)$ and $P_{N}\left(w_{1}, w_{2}\right)=p_{1}\left(w_{1}\right) p_{2}\left(w_{2}\right)$.

2. The gauge factor $\Psi_{0}\left(w_{1}, w_{2}\right)$. It plays the role of a generalized "ground state function". This non-polynomial function $\Psi_{0}\left(w_{1}, w_{2}\right)$ describes the asymptotic behavior of $\Psi$ in (1), i.e., it determines whether the solution is square integrable or not.

3. The general constraint. The operator $H$ in the original Laplace equation $H \Psi=0$ is gauge transformed to

$$
h \equiv \Psi_{0}^{-1} H \Psi_{0} .
$$

In general, the condition $H \Psi=0$ leads to an eigenvalue problem $h^{\prime} P=E P$, where $E=E\left(a_{1}, a_{2}, a_{3}, a_{4}\right)$ and $h^{\prime}=h-E$. Unlike $h$, the operator $h^{\prime}$ has no constant term. Moreover, the operator $h(4)$ maps polynomials into polynomials without increasing the order. Therefore, it can be written in terms of generators (2) where $N$ in (2) is determined by the parameters of the potential $V: N=N\left(a_{1}, a_{2}, a_{3}, a_{4}\right)$. Then, for a non-negative integer $N$ the operator $h(4)$ possesses a polynomial invariant subspace $\mathcal{P}_{N}$, i.e., in variables $w_{1}, w_{2}$. 


\subsection{Summary of relations between conformal Stäckel trans- forms and polynomial solutions of Laplace systems}

- Conformal Stäckel Transform (CST): Assume

$$
\begin{gathered}
H \Psi=\left(\partial_{x}^{2}+\partial_{y}^{2}+V(x, y)\right) \Psi=0 ; \quad V=V_{0}+\alpha U, \\
\text { metric : } d s^{2}=d x^{2}+d y^{2}, \quad \text { measure : } d x d y, \\
C S T: \quad \tilde{H} \Psi=\left(U^{-1}\left(\partial_{x}^{2}+\partial_{y}^{2}\right)+U^{-1} V_{0}+\alpha\right) \Psi=0, \\
\text { metric : } d s^{2}=U\left(d x^{2}+d y^{2}\right), \quad \text { measure : } U d x d y .
\end{gathered}
$$

- Polynomial solutions: Assume $H \Psi=0$ with $\Psi=\Psi_{0}(x, y) P\left(w_{1}, w_{2}\right)$ where $\Psi_{0}$ is the "ground state wave function" and $P$ is a polynomial in suitable independent variables $w_{j}(x, y), j=1,2$.

$$
\begin{gathered}
\left.H^{\prime} P=\left(\Psi_{0}^{-1}\left(\partial_{x}^{2}+\partial_{y}^{2}\right) \Psi_{0}+V_{0}+\alpha U\right)\right) P=0 \\
\Psi_{0}^{-1}\left(\partial_{x}^{2}+\partial_{y}^{2}\right) \Psi_{0}=\partial_{x}^{2}+\partial_{y}^{2}+2\left(\left(\partial_{x} \Psi_{0}\right) \partial_{x}+\left(\partial_{y} \Psi_{0}\right) \partial_{y}\right)+\left(\partial_{x}^{2} \Psi_{0}\right)+\left(\partial_{y}^{2} \Psi_{0}\right) . \\
\text { measure }:\left|\Psi_{0}\right|^{2} d x d y \\
C S T: \quad \tilde{H}^{\prime} P=\left(\left(\Psi_{0} U\right)^{-1}\left(\partial_{x}^{2}+\partial_{y}^{2}\right) \Psi_{0}+U^{-1} V_{0}+\alpha\right) P=0 \\
\text { metric : } d s^{2}=U\left(d x^{2}+d y^{2}\right), \quad \text { measure : }\left|\Psi_{0}\right|^{2} U d x d y
\end{gathered}
$$

\section{Non-degenerate potentials}

In this section we treat the 8 generic Laplace superintegrable systems with non-degenerate potential, listed in Table 1. Each of the 44 known Helmholtz non-degenerate superintegrable (i.e. 3-parameter) eigenvalue systems is Stäckel equivalent to exactly one of these generic systems, [2].

1. System [1111]:

This system is exactly solvable and is $R$-separable in 2 sets of coordinates

(a) Spherical : $x=\frac{\sin \theta \cos \phi}{1+\cos \theta}, y=\frac{\sin \theta \sin \phi}{1+\cos \theta}$,

(b) Elliptic: $\quad x^{2}=\frac{(c u-1)(c v-1)}{(1-c)(1+\sqrt{c u v})^{2}}, y^{2}=\frac{c(u-1)(v-1)}{(c-1)(1+\sqrt{c u v})^{2}}$, $c$ is a parameter $\neq 0,1$.

The polynomial variables are

$$
w_{1}=\frac{4 x^{2}}{\left(x^{2}+y^{2}+1\right)^{2}}, \quad w_{2}=\frac{4 y^{2}}{\left(x^{2}+y^{2}+1\right)^{2}} .
$$


Writing the function $\Psi\left(w_{1}, w_{2}\right)=\Psi_{0}\left(w_{1}, w_{2}\right) P\left(w_{1}, w_{2}\right)$ in (1) with the gauge factor $\Psi_{0}=w_{1}^{k_{1}} w_{2}^{k_{2}}\left(1-w_{1}-w_{2}\right)^{k_{3}}$, and

$$
a_{1}=-2 k_{1}\left(2 k_{1}-1\right), a_{2}=-2 k_{2}\left(2 k_{2}-1\right), a_{3}=-2 k_{3}\left(2 k_{3}-1\right),
$$

we arrive at the equation for the polynomial function $P\left(w_{1}, w_{2}\right)$ :

$$
\begin{gathered}
h^{(E S)} P \equiv\left[2 w_{1}\left(1-w_{1}\right) \partial_{w_{1}}^{2}+2 w_{2}\left(1-w_{2}\right) \partial_{w_{2}}^{2}-4 w_{1} w_{2} \partial_{w_{1}, w_{2}}^{2}\right. \\
\left.+\left(1+4 k_{1}-w_{1}(3+4 K)\right) \partial_{w_{1}}+\left(1+4 k_{2}-w_{2}(3+4 K)\right) \partial_{w_{2}}+E_{0}\right] P=0,
\end{gathered}
$$

where $K \equiv k_{1}+k_{2}+k_{3}$ and $E_{0}=-\frac{1}{2}\left[a_{4}+2 K(1+2 K)\right]$. Variables $w_{1}, w_{2}$ in (6) are not separated, but the operator $h^{(E S)}$ in (6) acting on $P$ maps polynomials, in these variables, into polynomials without increasing the order and is formally self-adjoint with respect to the inner product

$\left\langle P_{1}, P_{2}\right\rangle \equiv \iint d w_{1} d w_{2} P_{1}\left(w_{1}, w_{2}\right) \overline{P_{2}}\left(w_{1}, w_{2}\right)\left|\Psi_{0}\right|^{2} w_{1}^{-\frac{1}{2}} w_{2}^{-\frac{1}{2}}\left(1-w_{1}-w_{2}\right)^{-\frac{1}{2}}$.

Boundaries of the configuration space (domain), $w_{1} \geq 0, w_{2} \geq 0$ and $w_{1}+w_{2} \leq 1$, are determined by zeros of $\Psi_{0}$. Square-integrability demands $k_{1}, k_{2}, k_{3} \geq 1 / 4$.

Equation (6) can be rewritten in terms of $g l_{3}$ generators (2):

$$
\begin{gathered}
h^{(E S)} P=\left[2 \mathcal{J}_{11}^{0}\left(\mathcal{J}_{1}^{-}-\mathcal{J}_{11}^{0}\right)+\left(1+4 k_{1}\right) \mathcal{J}_{1}^{-}-(1+4 K) \mathcal{J}_{11}^{0}\right. \\
\left.+2 \mathcal{J}_{22}^{0}\left(\mathcal{J}_{2}^{-}-\mathcal{J}_{22}^{0}\right)+\left(1+4 k_{2}\right) \mathcal{J}_{2}^{-}-(1+4 K) \mathcal{J}_{22}^{0}-4 \mathcal{J}_{12}^{0}+E_{0}\right] P=0 .
\end{gathered}
$$

Thus the operator $h^{(E S)}$ has a hidden $g l_{3}$ algebra. Infinitely many finite-dimensional invariant subspaces (3) leads to the constraint

$$
2(N+K)(2 N+2 K+1)+a_{4}=0,
$$

By adding the trivial raising operator

$$
\mathcal{J}^{+}(N) \equiv \mathcal{J}_{1}^{+}(N)+\mathcal{J}_{2}^{+}(N)=\left(w_{1}+w_{2}\right)\left(w_{1} \partial_{w_{1}}+w_{2} \partial_{w_{2}}-N\right),
$$

the exactly-solvable operator $h^{(E S)}$ that annihilates $P$ can be easily generalized to a quasi-exactly-solvable one $h^{(Q E S)}=h^{(E S)}+\alpha \mathcal{J}^{+}(N)$, with real parameter $\alpha$. Now this operator has a single invariant subspace in 2 -variate polynomials. Moreover, with a term $I=\mathcal{J}_{1}^{-}-\mathcal{J}_{2}^{-}$, we can form the operator $h^{(P T)}$

$$
h^{(P T)}=h^{(E S)}+\alpha \mathcal{J}^{+}(N)+\beta I,
$$

where $\beta \neq 0$ is a parameter. The operator $h^{(P T)}$ is also quasi-exactlysolvable. However, unlike $h^{(E S)}(6), h^{(P T)}$ is not formally self-adjoint 
due to the boundary terms. For $\beta$ a pure complex number $h^{(P T)}$ is invariant under the operation of complex conjugation followed by the transposition $w_{1} \leftrightarrow w_{2}$. Thus the system is PT-symmetric, with real energy eigenvalues, [20].

The basis of conformal symmetries is given by the set $\left\{L_{1}, L_{2}, h^{(E S)}\right\}$ where

$$
\begin{aligned}
L_{1}= & w_{1} w_{2}\left(\partial_{w_{1}}^{2}-2 \partial_{w_{1} w_{2}}+\partial_{w_{2}}^{2}\right)+\left(\frac{w_{1}}{2}\left(1+4 k_{2}\right)-\frac{w_{2}}{2}\left(1+4 k_{1}\right)\right)\left(\partial_{w_{2}}-\partial_{w_{1}}\right), \\
L_{2}= & -w_{1}\left(w_{1}+w_{2}-1\right) \partial_{w_{1}}^{2}+ \\
& \left(\frac{1}{2}+2 k_{1}-w_{1}\left(1+2 k_{1}+2 k_{3}\right)-\frac{1}{2} w_{2}\left(1+4 k_{1}\right)\right) \partial_{w_{1}}-2 k_{1} .
\end{aligned}
$$

which also can be written in terms of $g l_{3}$ generators (2)

$$
\begin{gathered}
L_{1}=\mathcal{J}_{12}^{0} \mathcal{J}_{22}^{0}+\mathcal{J}_{21}^{0} \mathcal{J}_{11}^{0}-2 \mathcal{J}_{22}^{0} \mathcal{J}_{11}^{0}-\mathcal{J}_{12}^{0}-\mathcal{J}_{21}^{0} \\
+\frac{1+4 k_{2}}{2}\left(\mathcal{J}_{12}^{0}-\mathcal{J}_{11}^{0}\right)+\frac{1+4 k_{1}}{2}\left(\mathcal{J}_{21}^{0}-\mathcal{J}_{22}^{0}\right) \\
L_{2}=-\mathcal{J}_{21}^{0} \mathcal{J}_{11}^{0}-\mathcal{J}_{11}^{0} \mathcal{J}_{11}^{0}+\mathcal{J}_{11}^{0} \mathcal{J}_{1}^{-}+\mathcal{J}_{21}^{0}+\mathcal{J}_{11}^{0}+\left(\frac{1}{2}+2 k_{1}\right)\left(\mathcal{J}_{1}^{-}\right. \\
\left.-\mathcal{J}_{21}^{0}\right)-\left(1+2 k_{1}+2 k_{3}\right) \mathcal{J}_{11}^{0}-2 k_{1} .
\end{gathered}
$$

The solutions of (6) separable in spherical coordinates are products of Jacobi polynomials and are the eigenfunctions of $L_{1}$ while those separable in elliptic coordinates are eigenfunctions of $L_{1}+(1-r) L_{2}$ and can be factorized as the the product of two Heun polynomials.

Even though this is an exactly solvable system, the separation equations in elliptic coordinates are quasi-exactly-solvable. Indeed, setting $P(u, v)=$ $U(u) V(v)$ with the constraint (7) we find the separation equation

$$
\begin{gathered}
4 u(r u-1)(u-1) U^{\prime \prime}(u)+2\left((4 r K+3 r) u^{2}-\left(4 r K+2 r+2+4 k_{2}+4 k_{3}\right) u\right. \\
\left.+4 k_{3}+1\right) U^{\prime}(u)+2 r N(2 N+4 K+1) u U(u)=\Lambda U(u),
\end{gathered}
$$

with an exactly similar equation for $V(v)$, where $\Lambda$ is the separation parameter. These equations are QES; they have $N+1$ eigenvalues $\Lambda_{j}$ on the space of polynomials of maximum order $N$ in $u$ and $v$, respectively. For Helmholtz versions of these observations, see [18].

2. System [211]:

The system [211] corresponds to the well-known Smorodinsky-Winternitz potential [21]. It is $R$-separable in 3 sets of coordinates
(a) Cartesian: $\quad x, y$,
(b) Polar : $\quad x=r \cos \theta, y=r \sin \theta$,
(c) Elliptic: $\quad x=c \sqrt{(u-1)(v-1)}, y=c \sqrt{-u v}$, 
where $c \neq 0$ is a parameter. The polynomial variables are $w_{1}=x^{2}, w_{2}=y^{2}$, and the gauge factor reads $\Psi_{0}=\exp \left[k_{3}\left(w_{1}+w_{2}\right)\right] w_{1}^{k_{1}} w_{2}^{k_{2}}$. With

$$
a_{1}=-2 k_{1}\left(2 k_{1}-1\right), a_{2}=-2 k_{2}\left(2 k_{2}-1\right), a_{3}=4 k_{3}^{2},,
$$

and $\Psi\left(w_{1}, w_{2}\right)=\Psi_{0}\left(w_{1}, w_{2}\right) P\left(w_{1}, w_{2}\right)$ we obtain the equation

$$
\begin{gathered}
h^{(E S)} P \equiv\left[4 w_{1} \partial_{w_{1}}^{2}+4 w_{2} \partial_{w_{2}}^{2}+2\left(4 k_{1}+1+4 k_{3} w_{1}\right) \partial_{w_{1}}\right. \\
\left.+2\left(4 k_{2}+1+4 k_{3} w_{2}\right) \partial_{w_{2}}+4 k_{3}\left(1+2 k_{1}+2 k_{2}\right)+a_{4}\right] P=0 .
\end{gathered}
$$

Unlike the previous case (6), in this problem separation in the variables $w_{1}, w_{2}$ occurs. Factorization takes place and, essentially, we are left with two independent one-dimensional problems. The operator $h^{(E S)}$ maps polynomials, in variables $w_{1}, w_{2}$, into polynomials without increasing the order and is formally self-adjoint with respect to the inner product

$$
\left\langle P_{1}, P_{2}\right\rangle \equiv \iint d w_{1} d w_{2} P_{1}\left(w_{1}, w_{2}\right) \overline{P_{2}}\left(w_{1}, w_{2}\right)\left|\Psi_{0}\right|^{2} w_{1}^{-\frac{1}{2}} w_{2}^{-\frac{1}{2}} .
$$

Boundaries of the configuration space (domain), $w_{1} \geq 0, w_{2} \geq 0$ are determined by zeros and singularities of $\Psi_{0}$. Square-integrability demands $k_{1} \geq \frac{1}{4}, k_{2} \geq \frac{1}{4}, k_{3}<0$.

The basis of symmetries is $\left\{L_{1}, L_{2}, h^{(E S)}\right\}$ where

$$
\begin{aligned}
L_{1} & =2 w_{1} \partial_{w_{1}}^{2}+\left(4 k_{1}+1+4 k_{3} w_{1}\right) \partial_{w_{1}}+2 k_{3}\left(1+2 k_{1}\right), \\
L_{2} & =4 w_{1} w_{2}\left(\partial_{w_{1}}^{2}-2 \partial_{w_{1} w_{2}}+\partial_{w_{2}}^{2}\right)+\left(-8 k_{2} w_{1}+8 k_{1} w_{2}-2 w_{1}+2 w_{2}\right) \partial_{w_{1}}, \\
& +\left(8 k_{2} w_{1}-8 k_{1} w_{2}+2 w_{1}-2 w_{2}\right) \partial_{w_{2}} .
\end{aligned}
$$

The separation equations in Cartesian and polar coordinates are exactly solvable (products of associated Laguerre polynomials in the Cartesian case, products of associated Laguerre and Jacobi polynomials in the polar case); for elliptic coordinates they are QES (products of Heun polynomials). Indeed, setting $P(u, v)=U(u) V(v)$ with the constraint

$$
4 k_{3}\left(2 N+2 k_{1}+2 k_{2}+1\right)+a_{4}=0 .
$$

we find the separation equation

$$
\begin{gathered}
-4 u(u-1) U^{\prime \prime}(u)+2\left(4 k_{3} c^{2} u^{2}-2\left(2 k_{1}+2 k_{2}+2 k_{3}^{2}+1\right) u+4 k_{2}+1\right) U^{\prime}(u) \\
-8 k_{3} c^{2} N u U(u)=\Lambda U(u),
\end{gathered}
$$

with an exactly similar equation for $V(v)$, where $\Lambda$ is the separation parameter. These equations have $N+1$ eigenvalues $\Lambda_{j}$ on the space of polynomials of maximum order $N$ in $u$ and $v$, respectively.

In terms of the generators $\mathcal{J}$ 's (2) the equation (9) takes the form

$$
\begin{aligned}
h^{(E S)} P & \equiv\left[4 \mathcal{J}_{11}^{0} \mathcal{J}_{1}^{-}+8 k_{3}\left(\mathcal{J}_{11}^{0}+\mathcal{J}_{22}^{0}\right)+2\left(1+4 k_{1}\right) \mathcal{J}_{1}^{-}\right. \\
& \left.+4 \mathcal{J}_{22}^{0} \mathcal{J}_{2}^{-}+2\left(1+4 k_{2}\right) \mathcal{J}_{2}^{-}+E_{0}\right] P=0
\end{aligned}
$$


If the constraint (10) is satisfied then $h^{(E S)}$ admits a basis of polynomial solutions of highest total order $N=0,1,2, \ldots$ in variables $w_{1}, w_{2}$. The dependence of the spectral parameter $-a_{4}$ is linear in quantum number $N$.

Also, $h^{(E S)}$ becomes quasi-exactly-solvable by adding the raising operator $\mathcal{J}^{+}(N): h^{(Q E S)}=h^{(E S)}+\alpha \mathcal{J}^{+}(N)$, with parameter $\alpha$. The term $I=$ $\mathcal{J}_{1}^{-}-\mathcal{J}_{2}^{-}, h^{(P T)}=h^{(E S)}+\alpha \mathcal{J}^{+}(N)+\beta I$, with $\beta$ a parameter can be added as well and the system remains quasi-exactly-solvable. However, for $\beta \neq 0$, due to the boundary terms it is not formally self-adjoint anymore. For $\beta$ a pure complex number the operator $h^{(P T)}$ is invariant under the operation of complex conjugation followed by the transposition $w_{1} \leftrightarrow w_{2}$. Thus the system is PT-symmetric and the energy eigenvalues are real. For comparison, let us work out the explicit form of such a PT-symmetric operator.

The unitary equivalent operator $H^{(P T)}=\tilde{g} h^{(P T)} \tilde{g}^{-1}$, where $\tilde{g}=e^{-\alpha\left(w_{1}+w_{2}\right)^{2}} \Psi_{0}\left(w_{1}, w_{2}\right)$, written in Cartesian variables $(x, y)$ reads

$$
H^{(P T)}=H+\frac{\beta}{2}\left(\frac{1}{x} \partial_{x}-\frac{1}{y} \partial_{y}\right)+V_{N} .
$$

Here $H=\Delta_{2}+\frac{a_{1}}{x^{2}}+\frac{a_{2}}{y^{2}}-a_{3}\left(x^{2}+y^{2}\right)+a_{4}$ is the original operator we started with (systems [211]) and

$V_{N}=-\frac{\alpha\left(x^{2}+y^{2}\right)}{16}\left[4\left(6-k_{1}-k_{2}\right)+16 N-8 k_{3}\left(x^{2}+y^{2}\right)+\alpha\left(x^{2}+y^{2}\right)^{2}\right]$,

$a_{1}=\left(-k_{1}^{2}+1\right) / 4, a_{2}=\left(-k_{2}^{2}+1\right) / 4, a_{3}=k_{3}^{2}$. Unlike $H$, the operator $H^{(P T)}$ does not admit separation of variables.

3. System [22]:

This system is $R$-separable in 2 sets of coordinates

(a) Polar : $x=r \cos \theta, y=r \sin \theta$,

(b) Hyperbolic: $x=\frac{u^{2}+v^{2}+u^{2} v^{2}}{2 u v}, y=\frac{u^{2}+v^{2}-u^{2} v^{2}}{2 u v}$.

Strictly, this system is neither ES nor QES, though of a simple type where the polynomial solutions are explicit!

The polynomial variables are $w_{1}=x^{2}+y^{2}, w_{2}=(x-i y) /(x+i y)$. With the gauge factor $\Psi_{0}=e^{\left(k_{1} w_{1}+k_{2} w_{2}\right)}\left(w_{1} w_{2}\right)^{-k_{3}}, \Psi(x, y)=\Psi_{0} P\left(w_{1}, w_{2}\right)$, and $a_{1}=4 k_{2}\left(2 n_{2}-2 k_{3}+1\right), a_{2}=4 k_{2}^{2}, a_{3}=-4 k_{1}\left(2 n_{1}-2 k_{3}+1\right), a_{4}=4 k_{1}^{2}$, we have

$$
\begin{aligned}
& h^{(E S)} P \equiv\left[4 w_{1}^{2} \partial_{w_{1}}^{2}-4 w_{2}^{2} \partial_{w_{2}}^{2}+4 w_{1}\left(2 k_{1} w_{1}-2 k_{3}+1\right) \partial_{w_{1}}\right. \\
& \left.-4 w_{2}\left(2 k_{2} w_{2}-2 k_{3}+1\right) \partial_{w_{2}}+8\left(-k_{1} n_{1} w_{1}+k_{2} n_{2} w_{2}\right)\right] P=0 .
\end{aligned}
$$

Here, $n_{1}, n_{2}$ are nonnegative integers. The operator $h^{(E S)}$ maps polynomials of order $n_{1}$ in $w_{1}$ and $n_{2}$ in $w_{2}$ without increasing the order. A set of eigenfunctions of $L_{1}$ is

$$
f_{n}\left(w_{1}, w_{2}\right)=\left(w_{1} w_{2}\right)^{n} \times
$$




$$
{ }_{1} F_{1}\left(\begin{array}{c}
n-n_{1} \\
1+2\left(n-k_{3}\right)
\end{array} ;-2 k_{1} w_{1}\right){ }_{1} F_{1}\left(\begin{array}{c}
n-n_{2} \\
1+2\left(n-k_{3}\right)
\end{array} ;-2 k_{2} w_{2}\right),
$$

where $0 \leq n \leq n_{1}, n_{2}$ and $n$ is an integer.

The basis of conformal symmetries is given by $\left\{L_{1}, L_{2}, h^{(E S)}\right\}$

$$
\begin{aligned}
L_{1} & =-4 w_{2}^{2} \partial_{w_{2}}^{2}-4 w_{2}\left(2 k_{2} w_{2}-2 k_{3}+1\right) \partial_{w_{2}}-4\left(-k_{2} n_{2} w_{2}\right. \\
& \left.+\left(k_{2}-k_{3}\right)^{2}+2 k_{2} n_{2}+k_{2}\right), \\
L_{2} & =\frac{4 w_{1}}{w_{2}} \partial_{w_{1}}^{2}+8 \partial_{w_{1} w_{2}}+\frac{4 w_{2}}{w_{1}} \partial_{w_{2}}^{2}+\frac{8}{w_{2}}\left(k_{1} w_{1}+k_{2} w_{2}-2 k_{3}\right) \partial_{w_{1}} \\
& +\frac{8}{w_{1}}\left(k_{1} w_{1}+k_{2} w_{2}-2 k_{3}\right) \partial_{w_{2}}-\frac{8 k_{3}}{w_{1} w_{2}}\left(2 k_{1} w_{1}+2 k_{2} w_{2}-2 k_{3}-1\right) .
\end{aligned}
$$

Here, $L_{1}$ maps polynomials to polynomials, subject to the constraints listed above, but $L_{2}$ does not. This implies that polynomial solutions can be realized in polar coordinates (products of associated Laguerre polynomials), but not in hyperbolic coordinates (products of double confluent Heun functions).

For $\tau=\exp (-2 i \theta), P(r, \tau)=S(r) T(\tau)$ the separation equations in polar coordinates are

$$
\begin{gathered}
r^{2} S^{\prime \prime}(r)+r\left(4 k_{1} r^{2}+2 k_{3}-4 m-1\right) S^{\prime}(r)+8 k_{1} m r^{2} S(r)=\Lambda S(r), \\
4 \tau^{2} T^{\prime \prime}(\tau)+4 \tau\left(2 k_{2} \tau+k_{3}-2 m\right) T^{\prime}(\tau)-8 k_{2} m \tau T(\tau)=\Lambda T(\tau),
\end{gathered}
$$

where $\Lambda$ is the separation parameter. These equations have $m+1$ eigenvalues $\Lambda_{j}$ on the spaces of polynomials of maximum order $m$ in $r^{2}$ and $\tau$, respectively.

4. System [31]:

The system is exactly-solvable and $R$-separable in 2 sets of coordinates

$$
\begin{aligned}
& \text { (a) Cartesian : } x, y, \\
& \text { (b) Parabolic: } x=\xi^{2}-\eta^{2}, y=2 \xi \eta \text {. }
\end{aligned}
$$

The polynomial variables are $w_{1}=x, w_{2}=y^{2}$. The gauge factor is given by

$$
\Psi_{0}=e^{\left(k_{3}\left(w_{1}^{2}+\frac{w_{2}}{2}\right)+k_{2} w_{1}\right)} w_{2}^{k_{4}}, \quad \Psi(x, y)=\Psi_{0} P\left(w_{1}, w_{2}\right) .
$$

With $a_{2}=4 k_{2} k_{3}, a_{3}=-k_{3}^{2}, a_{4}=-2 k_{4}\left(2 k_{4}-1\right)$, we arrive at $h^{(E S)} P \equiv\left[\partial_{w_{1}}^{2}+4 w_{2} \partial_{w_{2}}^{2}+\left(2 k_{2}+4 k_{3} w_{1}\right) \partial_{w_{1}}+\left(8 k_{4}+2+4 k_{3} w_{2}\right) \partial_{w_{2}}\right.$

$$
\left.+k_{2}^{2}+4 k_{3} k_{4}+3 k_{3}+a_{1}\right] P=0 .
$$

In variables $w_{1}$ and $w_{2}$ the operator $h^{(E S)}$ maps 2-variate polynomials into polynomials without increasing the order and is formally self-adjoint with respect to the inner product

$$
\left\langle P_{1}, P_{2}\right\rangle \equiv \iint d w_{1} d w_{2} P_{1}\left(w_{1}, w_{2}\right) \overline{P_{2}}\left(w_{1}, w_{2}\right)\left|\Psi_{0}\right|^{2} w_{2}^{-\frac{1}{2}} .
$$


Boundaries of the configuration space (domain), $-\infty<w_{1}<\infty, 0 \leq w_{2}$ are determined by the zeros and singularities of $\Psi_{0}$. Square-integrability demands $k_{4} \geq \frac{1}{4}, k_{3}<0$. In terms of $g l_{3}$ generators $\mathcal{J}$ 's (2) the equation (13) reads

$$
\begin{gathered}
h^{(E S)} P=\left[\mathcal{J}_{1}^{-} \mathcal{J}_{1}^{-}+2 k_{2} \mathcal{J}_{1}^{-}+4 k_{3} \mathcal{J}_{11}^{0}+4 \mathcal{J}_{22}^{0} \mathcal{J}_{2}^{-}\right. \\
\left.+\left(8 k_{4}+2\right) \mathcal{J}_{2}^{-}+4 k_{3} \mathcal{J}_{22}^{0}+a_{1}+k_{2}^{2}+3 k_{3}+4 k_{3} k_{4}\right] P=0 .
\end{gathered}
$$

If $N$ is a non-negative integer with the constraint

$$
a_{1}+k_{3}\left(4 N+4 k_{4}+3\right)+k_{2}^{2}=0,
$$

then the operator $h^{(E S)}$ admits a basis of 2-variate polynomial solutions of highest total order $N$ in $w_{1}, w_{2}$.

The basis of symmetries is given by the operators $\left\{L_{1}, L_{2}, h^{(E S)}\right\}$

$$
\begin{aligned}
L_{1} & =\partial_{w_{1}}^{2}+\left(4 k_{3} w_{1}+2 k_{2}\right) \partial_{w_{1}}+\left(k_{2}^{2}+2 k_{3}\right), \\
L_{2} & =\left(-k_{3} w_{2}-2 k_{4}-\frac{1}{2}\right) \partial_{w_{1}}+\left(-2 k_{2} w_{2}+8 k_{4} w_{1}+2 w_{1}\right) \partial_{w_{2}} \\
& -2 w_{2} \partial_{w_{1} w_{2}}+w_{1} w_{2} \partial_{w_{2}}^{2}-\frac{1}{2} k_{2}\left(4 k_{4}+1\right) .
\end{aligned}
$$

These operators map polynomials to polynomials without increasing degree.

The separation equations in Cartesian coordinates are exactly solvable (products of Hermite and associated Laguerre polynomials); for parabolic coordinates they are QES (products of biconfluent Heun polynomials). Indeed, setting $P(u, v)=U(u) V(v)$ where $u=\xi^{2}, v=\eta^{2}$, and with the constraint mentioned above we find the separation equation

$$
\left.2 u U^{\prime \prime}(u)+\left(2 k_{3} u^{2}+2 k_{2} u+4 k_{4}+1\right)\right) U^{\prime}(u)-2 k_{3} N u U(u)=\Lambda U(u),
$$

with an exactly similar equation for $V(v)$, where $\Lambda$ is the separation constant. These equations have $N+1$ eigenvalues $\Lambda_{j}$ on the space of polynomials of maximum order $N$ in $\xi^{2}$ and $\eta^{2}$, respectively.

5. System [4]:

Similar to [22], this system is neither ES nor QES. It is $R$-separable only in the family of semi-hyperbolic coordinates, a representative of which is

$$
x=-(w-u)^{2}+i(w+u), \quad y=-i(w-u)^{2}+(w+u) .
$$

The polynomial variables are

$$
w_{1}=\frac{1}{4}(y-i x-i \sqrt{2(x-i y)}), w_{2}=\frac{1}{4}(y-i x+i \sqrt{2(x-i y)}) .
$$

Setting $a_{4}=-k_{4}^{2}$ and with gauge factor as

$$
\begin{gathered}
\Psi_{0}\left(w_{1}, w_{2}\right)= \\
\exp \left[\frac{1}{6 k_{4}^{3}}\left\{-32 k_{4}^{4}\left(w_{1}^{3}+w_{2}^{3}\right)+12 i k_{4}^{2} a_{3}\left(w_{1}^{2}+w_{2}^{2}\right)-3\left(k_{4}^{2} a_{2}+a_{3}^{2}\right)\left(w_{1}+w_{2}\right)\right\}\right],
\end{gathered}
$$


we obtain the equation

$$
\begin{aligned}
h^{(E S)} & P \equiv\left[-\left(\partial_{w_{1}}^{2}+\frac{\left(-32 k_{4}^{4} w_{1}^{2}+8 i k_{4}^{2} a_{3} w_{1}-k_{4}^{2} a_{2}-a_{3}^{2}\right)}{k_{4}^{3}} \partial_{w_{1}}\right)\right. \\
& +\left(\partial_{w_{2}}^{2}+\frac{\left(-32 k_{4}^{4} w_{2}^{2}+8 i k_{4}^{2} a_{3} w_{2}-k_{4}^{2} a_{2}-a_{3}^{2}\right)}{k_{4}^{3}} \partial_{w_{2}}\right) \\
& \left.-\frac{4 i}{k_{4}^{4}}\left(w_{1}-w_{2}\right)\left(8 i k_{4}^{5}-2 k_{4}^{4} a_{1}-k_{4}^{2} a_{2} a_{3}-a_{3}^{2}\right)\right] P=0 .
\end{aligned}
$$

For a non-negative integer $N=0,1,2, \ldots$, the operator $h^{(E S)}$ maps polynomials of order $\leq N$ in both $w_{1}$ and $w_{2}$ when

$$
a_{1}+4 i k_{4} N+\frac{a_{2} a_{3}}{2 k_{4}^{2}}+\frac{a_{3}^{2}}{2 k_{4}^{4}}-4 i k_{4}=0 .
$$

Note that $h^{(E S)}=Y_{1}-Y_{2}$ where

$$
Y_{s}=-\left(\partial_{w_{s}}^{2}+\frac{\left(-32 k_{4}^{4} w_{s}^{2}+8 i k_{4}^{2} a_{3} w_{s}-k_{4}^{2} a_{2}-a_{3}^{2}\right)}{k_{4}^{3}} \partial_{w_{1}}\right)+32 k_{4} N w_{s}
$$

for $s=1,2$. Now let $f$ be a polynomial eigenfunction of $Y_{s}$ of order $N$ (which must exist since the space of polynomials of order $\leq N$ in $w_{s}$ is invariant under $\left.Y_{s}\right): Y_{s} f\left(w_{s}\right)=\lambda f\left(w_{s}\right), s=1,2$, and set $P=f\left(w_{1}\right) f\left(w_{2}\right)$. Then

$$
\left.h^{(E S)} P=\left(Y_{1}-Y_{2}\right) f\left(w_{1}\right) f_{(} w_{2}\right)=(\lambda-\lambda) f\left(w_{1}\right) f\left(w_{2}\right)=0,
$$

There are $N+1$ eigenfunctions, (triconfluent Heun polynomials).

The basis of conformal symmetries consist of the operators $\left\{L_{1}, L_{2}, h^{(E S)}\right\}$,

$$
\begin{aligned}
L_{1} & =-\frac{1}{8\left(w_{1}-w_{2}\right)^{3} k_{4}^{5}}\left(16 w_{1}^{3} k_{4}^{6}-16 k_{4}^{6} w_{1}^{2} w_{2}-16 k_{4}^{6} w_{1} w_{2}^{2}\right. \\
& +16 w_{2}^{3} k_{4}^{6}\left(4 i a_{3} k_{4}^{4} w_{1}^{2}+8 i a_{3} k_{4}^{4} w_{1} w_{2}-4 i a_{3} k_{4}^{4} w_{2}^{2}+k_{4}^{5}\right) \partial_{w_{1}} \\
& +\frac{1}{8\left(w_{1}-w_{2}\right)^{3} k_{4}^{5}}\left(16 w_{1}^{3} k_{4}^{6}-16 k_{4}^{6} w_{1}^{2} w_{2}-16 k_{4}^{6} w_{1} w_{2}^{2}+16 w_{2}^{3} k_{4}^{6}\right. \\
& \left.-4 i a_{3} k_{4}^{4} w_{1}^{2}+8 i a_{3} k_{4}^{4} w_{1} w_{2}-4 i a_{3} k_{4}^{4} w_{2}^{2}+k_{4}^{5}\right) \partial_{w_{2}} \\
& +\frac{1}{16\left(w_{1}-w_{2}\right)^{2}} \partial_{w_{1}}^{2}-\frac{1}{8\left(w_{1}-w_{2}\right)^{2}} \partial_{w_{1} w_{2}}+\frac{1}{16\left(w_{1}-w_{2}\right)^{2}} \partial_{w_{2}}^{2}-\frac{a_{3}^{2}}{k_{4}^{2}}, \\
L_{2} & =\frac{w_{2}\left(-32 w_{1}^{2} k_{4}^{4}+8 i k_{4}^{2} a_{3} w_{1}-k_{4}^{2} a_{2}-a_{3}^{2}\right)}{\left(w_{1}-w_{2}\right) k_{4}^{3}} \partial_{w_{1}}- \\
& \\
& -\frac{w_{1}\left(-32 w_{2}^{2} k_{4}^{4}+8 i k_{4}^{2} a_{3} w_{2}-k_{4}^{2} a_{2}-a_{3}^{2}\right)}{\left(w_{1}-w_{2}\right) k_{4}^{3}} \partial_{w_{2}}+\frac{w_{2}}{\left(w_{1}-w_{2}\right)} \partial_{w_{1}}^{2} \\
& \frac{w_{1}}{\left(w_{1}-w_{2}\right)} \partial_{w_{2}}^{2}-\frac{1}{4 k_{4}^{6}}\left(16 i k_{4}^{5} a_{3}+\left(a_{3}^{4}+k_{4}^{4} a_{2}^{2}+2 k_{4}^{2} a_{2} a_{3}^{2}\right) .\right.
\end{aligned}
$$

Neither $L_{1}$ nor $L_{2}$ maps polynomials to polynomials. However, assuming constraint (15), $L_{2}$ maps polynomial solutions of $h^{(E S)} P=0$ with order $\leq N$ to solutions. Indeed

$$
L_{2}=J_{1}+\frac{2 k_{4}^{2} a_{2} a_{3}^{2}+k_{4}^{4} a_{2}^{2}+16 i k_{4}^{5} a_{3}+a_{3}^{4}}{4 k_{4}^{6}}+\frac{w_{1}}{w_{2}-w_{1}} h^{(E S)} .
$$


6. System [0]:

This system separates in a family of Cartesian coordinates. The polynomial variables are $w_{1}=x-\frac{a_{2}}{2 a_{4}}, w_{2}=y-\frac{a_{3}}{2 a_{4}}$. Note that in this case the polynomial coordinates depend on the parameters in the potential. With

$$
a_{2}=4 k_{4} k_{2}, a_{3}=4 k_{4} k_{3}, a_{4}=-4 k_{4}^{2}, \quad \Psi_{0}=\exp \left[k_{4}\left(w_{1}^{2}+w_{2}^{2}\right)\right]
$$

and $\Psi(x, y)=\Psi_{0}\left(w_{1}, w_{2}\right) P\left(w_{1}, w_{2}\right)$, we have

$h^{(E S)} P \equiv\left(\partial_{w_{1}}^{2}+\partial_{w_{2}}^{2}+4 k_{4} w_{1} \partial_{w_{1}}+4 k_{4} w_{2} \partial_{w_{2}}+k_{2}^{2}+k_{3}^{2}+a_{1}+4 k_{4}\right) P=0$.

The operator $h^{(E S)}$ is exactly-solvable, it maps polynomials into polynomials without increasing the order and is formally self-adjoint with respect to the inner product

$$
\left\langle P_{1}, P_{2}\right\rangle \equiv \iint d w_{1} d w_{2} P_{1}\left(w_{1}, w_{2}\right) \overline{P_{2}}\left(w_{1}, w_{2}\right)\left|\Psi_{0}\right|^{2} .
$$

The boundaries of the configuration space (domain) are $-\infty<w_{1,2}<\infty$. Square-integrability demands $k_{4}<0$. In terms of the $g l_{3}$ generators $\mathcal{J}$ 's,$(2)$ :

$h^{(E S)} P=\left[\mathcal{J}_{1}^{-} \mathcal{J}_{1}^{-}+\mathcal{J}_{2}^{-} \mathcal{J}_{2}^{-}+4 k_{4}\left(\mathcal{J}_{1}^{0}+\mathcal{J}_{2}^{0}\right)+k_{2}^{2}+k_{3}^{2}+a_{1}+4 k_{4}\right] P=0$.

If $4 k_{4} N+k_{2}^{2}+k_{3}^{2}+a_{1}+4 k_{4}=0$, then $h^{(E S)}$ admits a basis of polynomial solutions of highest total order $N$ in $w_{1}, w_{2}$. The basis of symmetries is given by $\left\{L_{1}, L_{2}, h^{(E S)}\right\}$ :

$$
\begin{aligned}
& L_{1}=4 k_{4} w_{1} \partial_{w_{1}}+\partial_{w_{1}}^{2}+k_{2}^{2}+2 k_{4} \\
& L_{2}=2 k_{4} w_{2} \partial_{w_{1}}+2 k_{4} w_{1} \partial_{w_{2}}+\partial_{w_{1} w_{2}}+k_{2} k_{3} .
\end{aligned}
$$

7. System (1):

This exceptional conformally superintegrable system is not exactly or quasiexactly solvable. However, if we assume factorizable solutions of the form $\Psi(x, y)=\exp (\lambda \bar{z}) \psi(z)$ for $z=x+i y, \bar{z}=x-i y$, then for $\Psi=\exp (\lambda \bar{z}) \Theta(z) P(z)$ with $\Theta=\exp \left[\frac{1}{4 \lambda}\left(\frac{a_{1}}{z}-\frac{a_{3}}{2 z^{2}}+\frac{a_{4}}{3 z^{3}}\right)\right]$, we have $\left(4 \lambda \frac{d}{d z}+a_{2}\right) P(z)=0$. The operator $\left(4 \lambda \frac{d}{d z}+a_{2}\right)$ takes polynomials in $z$ to polynomials, without increasing the degree. For $4 \lambda N+a_{2}=0$ the operator $\left(4 \lambda \frac{d}{d z}+a_{2}\right)$ admits a basis of polynomial solutions of highest total order $N$ in $z$.

The basis of symmetries is $\left\{L_{1}, L_{2}, H\right\}$ :

$$
\begin{aligned}
& L_{1}=\partial_{x}+i \partial_{y} \\
& L_{2}=\left\{x \partial_{y}-y \partial_{x}, \partial_{x}+i \partial_{y}\right\}+i\left(\frac{2 a_{1}}{(x+i y)}-\frac{3 a_{3}}{2(x+i y)^{2}}+\frac{4 a_{4}}{3(x+i y)^{3}}\right)
\end{aligned}
$$

8. System (2): As with the preceding case, this exceptional conformally superintegrable system is neither exactly nor quasi-exactly solvable. However, if we assume solutions of the form $\Psi(x, y)=\exp (\lambda \bar{z}) \psi(z)$ for $z=x+i y, \bar{z}=x-i y$, then for $\Psi=\exp (\lambda \bar{z}) \Theta(z) P(z)$ with $\Theta=\exp \left[-\frac{1}{4 \lambda}\left(\frac{a_{2} z^{2}}{2}+\frac{a_{3} z^{3}}{3}+\frac{a_{4} z^{4}}{4}\right)\right]$ 
we have $\left(4 \lambda \frac{d}{d z}+a_{1}\right) P(z)=0$. The operator $\left(4 \lambda \frac{d}{d z}+a_{1}\right)$ takes polynomials in $z$ to polynomials, without increasing the degree.

In this case the basis of symmetries is $\left\{L_{1}, L_{2}, H\right\}$

$$
\begin{aligned}
& L_{1}=\partial_{x}+i \partial_{y}, \\
& L_{2}=\left\{x \partial_{y}-y \partial_{x}, \partial_{x}+i \partial_{y}\right\}+i\left(\frac{a_{2}(x+i y)^{2}}{2}+\frac{2 a_{3}(x+i y)^{3}}{3}+\frac{3 a_{4}(x+i y)^{4}}{4}\right) .
\end{aligned}
$$

\section{Degenerate potentials}

In this section we consider Laplace superintegrable systems with two-parameter potentials. In total there are six degenerate potentials, listed in Table 2. To distinguish them from the non-degenerate ones hereafter the polynomial variables $\left(w_{1}, w_{2}\right)$ will be denoted as $\left(u_{1}, u_{2}\right)$. The two-parameter potentials can all be obtained as parameter restrictions of the previous four-parameter potentials, though they have additional symmetry.

\section{System A}

This system is ES, and $R$-separable in two coordinate systems:

(a) Spherical : $u_{1}=\sin \theta \cos \phi, u_{2}=\sin \theta \sin \phi$,

(b) Elliptic : $u_{1}^{2}=\frac{(c u-1)(c v-1)}{(1-c)}, u_{2}^{2}=\frac{c(u-1)(v-1)}{(c-1)}$, $c$ is a parameter $\neq 0,1$,

The polynomial variables are $u_{1}=\frac{2 x}{x^{2}+y^{2}+1}, u_{2}=\frac{2 y}{x^{2}+y^{2}+1}$. With $a_{3}=$ $-2 k_{3}\left(2 k_{3}-1\right), \Psi_{0}=\left(1-u_{1}^{2}-u_{2}^{2}\right)^{k_{3}}$, and $\Psi\left(u_{1}, u_{2}\right)=\Psi_{0} P\left(u_{1}, u_{2}\right)$, we have

$$
\begin{gathered}
h^{(E S)} P \equiv\left[\left(1-u_{1}^{2}\right) \partial_{u_{1}}^{2}+\left(1-u_{2}^{2}\right) \partial_{u_{2}}^{2}-2 u_{1} u_{2} \partial_{u_{1} u_{2}}^{2}\right. \\
\left.-u_{1}\left(2+4 k_{3}\right) \partial_{u_{1}}-u_{2}\left(2+4 k_{3}\right) \partial_{u_{2}}-\left(2 k_{3}\left(2 k_{3}+1\right)+a_{4}\right)\right] P=0 .
\end{gathered}
$$

The operator $h^{(E S)}$ acting on $P\left(u_{1}, u_{2}\right)$ maps polynomials into polynomials without increasing the order and is formally self-adjoint with respect to the inner product

$$
\left\langle P_{1}, P_{2}\right\rangle \equiv \iint d u_{1} d u_{2} P_{1}\left(u_{1}, u_{2}\right) \overline{P_{2}}\left(u_{1}, u_{2}\right)\left|\Psi_{0}\right|^{2}\left(1-u_{1}^{2}-u_{2}^{2}\right)^{-\frac{1}{2}} .
$$

The boundaries of the configuration space (domain) $u_{1} \geq 0, u_{2} \geq 0$ and $u_{1}^{2}+u_{2}^{2} \leq 1$ corresponds to the zeros of $\Psi_{0}$. Square-integrability demands $k_{3} \geq \frac{1}{4}$. 
In terms of generators $\mathcal{J}^{‘} \mathrm{~s},(2)$,

$$
\begin{gathered}
h^{(E S)} P=\left[\mathcal{J}_{1}^{-} \mathcal{J}_{1}^{-}+\mathcal{J}_{2}^{-} \mathcal{J}_{2}^{-}-\left(\mathcal{J}_{1}^{0} \mathcal{J}_{1}^{0}+\mathcal{J}_{2}^{0} \mathcal{J}_{2}^{0}\right)-\left(1+4 k_{3}\right)\left(\mathcal{J}_{1}^{0}+\mathcal{J}_{2}^{0}\right)\right. \\
\left.-2 \mathcal{J}_{1}^{0} \mathcal{J}_{2}^{0}-\left(2 k_{3}\left(2 k_{3}+1\right)+a_{4}\right)\right] P=0 .
\end{gathered}
$$

There are infinitely many finite-dimensional invariant subspaces $\mathcal{P}_{M}^{(2)}=$ $\left\langle u_{1}^{p_{1}} u_{2}^{p_{2}} \mid 0 \leq p_{1}+p_{2} \leq M\right\rangle$ where $M=0,1,2 \ldots$, provided

$$
\left(M+2 k_{3}\right)\left(M+2 k_{3}+1\right)+a_{4}=0 .
$$

Again, by adding the raising operator

$$
\mathcal{J}^{+}(M)=\left(u_{1}+u_{2}\right)\left(u_{1} \partial_{u_{1}}+u_{2} \partial_{u_{2}}-M\right),
$$

the exactly-solvable operator $h^{(E S)}$ that annihilates $P\left(u_{1}, u_{2}\right)$ becomes quasi-exactly- solvable $h^{(Q E S)}=h^{(E S)}+\alpha \mathcal{J}^{+}(M)$, where $\alpha$ is a real parameter. The operator $h^{(Q E S)}$ has a single invariant subspace in 2 -variate polynomials. By adding the term

$$
I=\mathcal{J}_{1}^{-}-\mathcal{J}_{2}^{-}, \quad h^{(P T)}=h^{(E S)}+\alpha \mathcal{J}^{+}(M)+\beta I,
$$

with $\beta$ a parameter, the operator $h^{(P T)}$ is quasi-exactly-solvable. However, it is not formally self-adjoint due to the boundary terms. For $\beta$ a pure complex number, $h^{(P T)}$ is invariant under the operation of complex conjugation followed by the transposition $u_{1} \leftrightarrow u_{2}$. Thus the system is PT-symmetric and the energy eigenvalues are real.

For conformal symmetries we can take the basis $\left\{L_{1}=J^{2}, L_{2}, L_{3}, H\right\}$,

$$
\begin{gathered}
J=u_{1} \partial_{u_{2}}-u_{2} \partial_{u_{1}}, L_{2}=-\frac{1}{4}\left(u_{1}^{2}+u_{2}^{2}-1\right) \partial_{u_{1}}^{2}-\left(\frac{1}{4}+k_{3}\right) u_{1} \partial_{u_{1}}, \\
L_{3}=-2\left(u_{1}^{2}+u_{2}^{2}-1\right) \partial_{u_{1} u_{2}}-\left(1+4 k_{3}\right)\left(u_{2} \partial_{u_{1}}+u_{1} \partial_{u_{2}}\right) .
\end{gathered}
$$

Solutions $P\left(u_{1}, u_{2}\right)$ are separable in spherical coordinates (16). They are products of Jacobi polynomials corresponding to eigenfunctions of $L_{1}$; those separable in elliptic coordinates (17) are eigenfunctions of $-\frac{1}{4} L_{1}+(r-1) L_{2}$ and take the form of products of Heun polynomials. Indeed, in elliptic coordinates the conformal symmetry operator

$$
\begin{gathered}
K \equiv-\frac{1}{4} L_{1}+(r-1) L_{2}-\frac{1}{4}(r v-1) H \\
=v(v-1)(r v-1) \partial_{v v}+\left(\frac{1}{2}-(1+r)\left(2 k_{3}+1\right) v+r\left(2 k_{3}+\frac{3}{2}\right) v^{2}\right) \partial_{v} \\
+\frac{1}{4} r\left(4 k_{3}^{2}+2 k_{3}+a_{4}\right) v-\frac{1}{4}\left(4 k_{3}^{2}+2 k_{3}+a_{4}\right),
\end{gathered}
$$

is QES. For $a_{4}+2\left(N+k_{3}\right)\left(2 N+2 k_{3}+1\right)=0$, (i.e., $\left.M=2 N\right)$ maps the space of polynomials in $v$ with maximum order $N$ into itself. 


\section{System B}

This system is $R$-separable in four coordinate systems

(a) Cartesian : $x, y$,

(b) Polar : $x=r \cos \theta, y=r \sin \theta$.

(c) Parabolic : $x=\xi \nu, y=\frac{1}{2}\left(\xi^{2}-\nu^{2}\right)$,

(d) Elliptic: $x^{2}=c^{2}(u-1)(v-1), y^{2}=-c^{2} u v$, $c$ is a parameter $\neq 0$.

The polynomial variables are $u_{1}, u_{2}$ where $x^{2}=u_{1} u_{2}, y=\frac{1}{2}\left(u_{1}-\right.$ $\left.u_{2}\right)$. Setting

$a_{4}=A^{2}, a_{1}=-(N+2)\left(N+\frac{1}{2}\right), \Psi_{0}=\exp \left[\frac{i A}{2}\left(u_{1}+u_{2}\right)\right]\left(u_{1} u_{2}\right)^{-N / 2-1 / 4}$,

and $\Psi=\Psi_{0}\left(u_{1}, u_{2}\right) P\left(u_{1}, u_{2}\right)$, we have

$$
\begin{gathered}
\frac{1}{4}\left(u_{1}+u_{2}\right) h^{(E S)} P \equiv \\
{\left[\left(u_{1} \partial_{u_{1}}^{2}+u_{2} \partial_{u_{2}}^{2}+i\left(A u_{1}+i N\right) \partial_{u_{1}}+i\left(A u_{2}+i N\right) \partial_{u_{2}}-i A N\right] P=0 .\right.}
\end{gathered}
$$

In terms of generators $\mathcal{J}$ 's $(2)$

$$
\left[\mathcal{J}_{1}^{0} \mathcal{J}_{1}^{-}+\mathcal{J}_{2}^{0} \mathcal{J}_{2}^{-}+i A\left(\mathcal{J}_{1}^{0}+\mathcal{J}_{2}^{0}\right)-N\left(\mathcal{J}_{1}^{-}+\mathcal{J}_{2}^{-}\right)-i A N\right] \quad P=0 .
$$

This equation admits a basis of polynomial solutions of highest total order $N$ in $u_{1}, u_{2}$, provided $N$ is a non-negative integer. In this case the functions $\{\Psi\}$ are not square integrable in the domain $u_{1}, u_{2} \in[0, \infty)$.

The generating symmetries are $\left\{P_{y}, L_{1}, L_{2}, H\right\}$ where

$$
\begin{gathered}
P_{y}=\partial_{y}, \quad L_{1}=x^{2} \partial_{y y}-2 x y \partial_{x y}+y^{2} \partial_{x x}-x \partial_{x}-y \partial_{y}+\frac{a_{1} y^{2}}{x^{2}} \\
L_{2}=x \partial_{x y}-y \partial_{x x}+\frac{1}{2} \partial_{y}-\frac{a_{1} y}{x^{2}} .
\end{gathered}
$$

Solutions separable in Cartesian coordinates are eigenfunctions of $P_{y}^{2}$, and $P$ is a product of a Bessel function (not a polynomial) and a monomial. Those separable in polar coordinates are eigenfunctions of $L_{1}$, and $P$ is a product of a Bessel function (not a polynomial) and an associated Laguerre function. Those separable in parabolic coordinates are eigenfunctions of $L_{2} ; P$ is a product of two confluent hypergeometric functions. Those separable in elliptic coordinates are eigenfunctions of $L_{1}-c^{2} P_{y}^{2} ; P$ is a product of two spheroidal wave functions. 


\section{System C}

This system, the harmonic oscillator, is $R$-separable in coordinate systems

(a) Cartesian : $x, y$,

(b) Polar : $x=r \cos \theta, y=r \sin \theta$.

(c) Hyperbolic : $x=\frac{U^{2}+V^{2}+U^{2} V^{2}}{2 U V}, y=i \frac{U^{2}+V^{2}-U^{2} V^{2}}{2 U V}$,

(d) Elliptic: $x^{2}=c^{2}(u-1)(v-1), y^{2}=-c^{2} u v$, $c$ a parameter $\neq 0$.

The polynomial variables are $u_{1}=x^{2}, u_{2}=y^{2}$. Because of the $Z_{2}$ symmetry, the null space of $H$ splits into even and odd functions. Correspondingly, there are two possible gauge transformations. Putting $a_{4}=-k_{4}^{2}$, the first gauge transformations is $\Psi_{0,1}=\exp \left[k_{4}\left(x^{2}+y^{2}\right)\right]$. With $\Psi(x, y)=\Psi_{0,1} P\left(u_{1}, u_{2}\right)$ we have

$$
\begin{gathered}
h^{(E S)} P \equiv \\
{\left[\left(2+8 k_{4} u_{1}\right) \partial_{u_{1}}+\left(2+8 k_{4} u_{2}\right) \partial_{u_{2}}+4 u_{2} \partial_{u_{2}}^{2}+4 u_{1} \partial_{u_{1}}^{2}+\left(4 k_{4}+a_{1}\right)\right] P=0 .}
\end{gathered}
$$

If $a_{1}+4 k_{4}(2 N+1)=0$ and $N$ is a nonnegative integer then $h^{(E S)}$ leaves invariant the space of polynomials in $u_{1}, u_{2}$ of maximum order $N$. The second transformation is

$$
\Psi_{0,2}=\exp \left[k_{4}\left(x^{2}+y^{2}\right)\right] x y, \quad \Psi(x, y)=\Psi_{0,2} P\left(u_{1}, u_{2}\right),
$$

and the corresponding equation takes the form

$$
\begin{gathered}
h^{(E S)} P \equiv \\
{\left[\left(6+8 k_{4} u_{1}\right) \partial_{u_{1}}+\left(6+8 k_{4} u_{2}\right) \partial_{u_{2}}+4 u_{2} \partial_{u_{2}}^{2}+4 u_{1} \partial_{u_{1}}^{2}+\left(12 k_{4}+a_{1}\right)\right] P=0 .}
\end{gathered}
$$

If $a_{1}+4 k_{4}(2 M+3)=0$ and $M$ is a nonnegative integer then $h^{(E S)}$ also leaves invariant the space of polynomials in $u_{1}, u_{2}$ of maximum order $M$. For both $\Psi_{0,1}$ and $\Psi_{0,2}$, the corresponding operator $h^{(E S)}$ is exactly-solvable and is formally self-adjoint with respect to the inner product

$$
\left\langle P_{1}, P_{2}\right\rangle \equiv \iint d w_{1} d w_{2} P_{1}\left(w_{1}, w_{2}\right) \overline{P_{2}}\left(w_{1}, w_{2}\right)\left|\Psi_{0,1(2)}\right|^{2} .
$$

The configuration space is $w_{1} \geq 0, w_{2} \geq 0$.

The operators $\left\{L, L_{1}, L_{2}, H\right\}$

$$
L=x \partial_{y}-y \partial_{x}, L_{1}=\partial_{x}^{2}-4 k_{4}^{2} x^{2}, L_{2}=\partial_{x y}-4 k_{4}^{2} x y .
$$


form a basis of symmetries. Solutions separable in Cartesian coordinates are eigenfunctions of $L_{1}, P$ is a product of Hermite functions. Those separable in polar coordinates are eigenfunctions of $L^{2}$, and $P$ is an associated Laguerre function times a monomial. Solutions separable in hyperbolic coordinates are eigenfunctions of $L_{1}+i L_{2}+\frac{1}{2} L^{2}$, and $P$ is a product of Whittaker functions. Those solutions separable in elliptic coordinates are eigenfunctions of $L_{1}+c^{-2} L^{2}$ and $P$ is a product of confluent Heun functions. The polynomial spaces do not admit separated solutions in hyperbolic coordinates.

\section{System D}

This system is $R$-separable in two coordinate systems.

$$
\begin{aligned}
& \text { (a) Cartesian : } x, y, \\
& \text { (b) Parabolic : } y=\xi \nu, x=\frac{1}{2}\left(\xi^{2}-\nu^{2}\right) .
\end{aligned}
$$

In this case there are no polynomial invariant subspaces. The generating symmetries of $H$ are $\left\{P_{y}, L_{1}, L_{2}, H\right\}$ where

$$
P_{y}=\partial_{y}, L_{1}=\frac{1}{2}\left\{x \partial_{y}-y \partial_{x}, \partial_{y}\right\}+\frac{a_{2}}{4} y^{2}, L_{2}=\partial_{x y}-\frac{a_{2}}{2} y .
$$

Solutions separable in Cartesian coordinates are eigenfunctions of $P_{y}^{2}$, they are given by the product of an Airy function times an exponential. Those separable in parabolic coordinates are eigenfunctions of $L_{1}$, they factorize as the product of triconfluent Heun functions.

\section{System E}

This is $R$-separable in two coordinate systems.

(a) $\quad$ polar : $x=r \cos \theta, y=r \sin \theta$,

(b) hyperbolic :

$$
x=\frac{U^{2}+V^{2}+U^{2} V^{2}}{2 U V}, y=i \frac{U^{2}+V^{2}-U^{2} V^{2}}{2 U V} .
$$

As for the system $D$, in this case there are no polynomials invariant subspaces. The generating symmetries of $H$ are $\left\{P_{+}, L_{1}, L_{2}, H\right\}$ where

$$
P_{+}=\partial_{x}+i \partial_{y}, L_{1}=\frac{1}{2}\left\{M, P_{+}\right\}-i \frac{a_{1}}{(x+i y)}, L_{2}=M^{2}+a_{1} \frac{(x-i y)}{(x+i y)},
$$

with $M=x \partial_{y}-y \partial_{x}$. Solutions, product of Bessel functions, separable in polar coordinates are eigenfunctions of $L_{2}$. Those separable in hyperbolic coordinates are products of doubleconfluent Heun functions corresponding to the eigenfunctions of $L_{2}+P_{+}^{2}$. 
6. System $\mathbf{F}$ This system is $R$-separable in two coordinate systems.

(a) Cartesian : $x, y$,

(b) semi - hyperbolic :

$$
x=i(w-u)^{2}+2 i(w+u), y=-(w-u)^{2}+2(w+u) .
$$

The polynomial variables are $u_{1}=u, u_{2}=w$, the semi-hyperbolic coordinates. Setting $a_{2}=i \frac{k_{1}^{2}}{16}, a_{1}=\frac{k_{1} k_{4}}{4}$, together with the gauge factor

$\Psi_{0}=\exp \left[k_{1}\left(u^{2}+w^{2}\right)+k_{4}(u+w)\right]=\exp \left[\frac{k_{1}}{32}(y-i x)^{2}-\frac{k_{1}}{4}(y+i x)+\frac{k_{4}}{2}(y-i x)\right]$,

$\Psi=\Psi_{0} P(u, w)$, we obtain

$$
\begin{gathered}
8(u-w) h^{(E S)} P \equiv \\
{\left[\left(2 k_{1} u+k_{4}\right) \partial_{u}-\left(2 k_{1} w+k_{4}\right) \partial_{w}+\frac{1}{2} \partial_{u}^{2}-\frac{1}{2} \partial_{w}^{2}\right] P=0 .}
\end{gathered}
$$

In terms of generators $\mathcal{J}^{\text {‘s }}$ (2),

$$
\left[\frac{1}{2}\left(\mathcal{J}_{u}^{-} \mathcal{J}_{u}^{-}-\mathcal{J}_{w}^{-} \mathcal{J}_{w}^{-}\right)+2 k_{1}\left(\mathcal{J}_{u}^{0}-\mathcal{J}_{w}^{0}\right)+k_{4}\left(\mathcal{J}_{u}^{-}-\mathcal{J}_{w}^{-}\right)\right] P=0 .
$$

For any non-negative integer $N$ this equation admits a basis of polynomial solutions of highest order $N$ in $u$ and highest order $N$ in $w$. This space of polynomial variables does not admit separated solutions in Cartesian coordinates.

The generating symmetries are $\left\{P_{+}, L_{1}, L_{2}, H\right\}$ where

$$
\begin{gathered}
P_{+}=\partial_{x}+i \partial_{y}, \quad L_{1}=\partial_{x}^{2}-a_{2} x \\
L_{2}=(x-i y) \partial_{x y}+i x \partial_{y y}-y \partial_{x x}+\frac{1}{2} \partial_{y}-\frac{i}{2} \partial_{x}-\frac{i}{4} a_{2}(x-i y)^{2} .
\end{gathered}
$$

Solutions separable in Cartesian coordinates are eigenfunctions of $L_{1}$, they are given by a product of Airy functions. Those separable in semi-hyperbolic coordinates are eigenfunctions of $P_{+}^{2}-4 L_{1}-L_{2}$, i.e., they are products of parabolic cylinder functions.

\subsection{Polynomial solutions and conformal Stäckel transform}

For a given Laplace system our approach allows us to study in a unified way the whole family of equivalent Helmholtz systems at once. To illustrate how this general method works we study in some detail the Laplace system [211]. In particular, we will show that constraint (10) encodes the eigenvalues of 
all Helmholtz systems conformally Stäckel equivalent to [211]. Moreover, the polynomial solutions are the same.

We start from the equation

$$
H_{211} \Psi \equiv\left(\Delta_{2}+\frac{a_{1}}{x^{2}}+\frac{a_{2}}{y^{2}}-a_{3}\left(x^{2}+y^{2}\right)+a_{4}\right) \Psi=0,
$$

where $-a_{4}$ plays the role of the spectral parameter for the associated Helmholtz system

$$
H_{E 1} \Psi \equiv\left(\Delta_{2}+\frac{a_{1}}{x^{2}}+\frac{a_{2}}{y^{2}}-a_{3}\left(x^{2}+y^{2}\right)\right) \Psi=-a_{4} \Psi .
$$

From (10) we already know that $H_{211}$ possesses polynomials solutions for $a_{4}=4 k_{3}\left(2 N+2 k_{1}+2 k_{2}+1\right)$, where $a_{1}=-2 k_{1}\left(2 k_{1}-1\right), a_{2}=-2 k_{2}\left(2 k_{2}-\right.$ $1), a_{3}=4 k_{3}^{2}$. Now, let us consider the Stäckel equivalent system [E16], [8]. The corresponding Helmholtz equation is the flat space system

$$
H_{E 16} \Psi \equiv \frac{1}{x^{2}+y^{2}}\left(\Delta_{2}+\frac{a_{1}}{x^{2}}+\frac{a_{2}}{y^{2}}+a_{4}\right) \Psi=a_{3} \Psi,
$$

where the roles of $-a_{4}$ and $a_{3}$ are interchanged. In this case $a_{3}$ plays the role of the spectral parameter while the other parameters $a_{1}, a_{2}$ and $a_{4}$ remain fixed. The operator $H_{E 16}$ is formally self-adjoint with respect to the inner product

$$
\left\langle\Psi_{1}, \Psi_{2}\right\rangle \equiv \iint\left(x^{2}+y^{2}\right) d x d y \Psi_{1} \overline{\Psi_{2}} .
$$

The domain is $-\infty<x<\infty,-\infty<y<\infty$. Unlike the [211] system, now the measure in the inner product contains the extra factor $\left(x^{2}+y^{2}\right)$. From the constraint we have $k_{3}=a_{4} /\left[4\left(2 N+2 k_{1}+2 k_{2}+1\right)\right.$ so polynomial solutions of the Stäckel equivalent system [E16] (26) occur at quantized values of the spectral parameter $a_{3}$ given by

$$
a_{3}=\frac{a_{4}^{2}}{4\left(1+2\left(k_{1}+k_{2}+N\right)\right)^{2}} .
$$

Note that the dependence of the energy eigenvalue on $N$ is nonlinear.

We introduce parabolic coordinates $u=\frac{x^{2}-y^{2}}{2}, v=x y$. In these coordinates the polynomial variables are

$$
\begin{gathered}
w_{1}=\sqrt{u^{2}+v^{2}}+u, w_{2}=\sqrt{u^{2}+v^{2}}-u, \\
\Psi\left(w_{1}, w_{2}\right)=\Psi_{0}\left(w_{1}, w_{2}\right) P\left(w_{1}, w_{2}\right), \quad \Psi_{0}=w_{1}^{k_{1}} w_{2}^{k_{2}} e^{k_{3}\left(w_{1}+w_{2}\right)} .
\end{gathered}
$$

The operator $h^{(E S)}$ obtained from $H_{E 16}$ via the gauge transformation is formally self-adjoint with respect to the inner product

$$
\left\langle P_{1}, P_{2}\right\rangle \equiv \iint d w_{1} d w_{2} P_{1}\left(w_{1}, w_{2}\right) \overline{P_{2}}\left(w_{1}, w_{2}\right)\left|\Psi_{0}\right|^{2}\left(w_{1}+w_{2}\right) w_{1}^{-\frac{1}{2}} w_{2}^{-\frac{1}{2}} .
$$


Again, boundaries of the configuration space (domain), $w_{1} \geq 0, w_{2} \geq 0$, are determined by zeros and singularities of $\Psi_{0}$. Polynomial solutions of highest total order $N=0,1,2, \ldots$ in $w_{1}, w_{2}$ appear when the constraint is satisfied.

As a second example, we present the Helmholtz system $S 4$ on the sphere, conformally Stäckel equivalent to $[2,1,1]$, for which the Laplace equation takes the form

$$
\left(\frac{x^{2} y^{2}}{x^{2}+y^{2}} \Delta_{2}-a_{3} x^{2} y^{2}+a_{4} \frac{x^{2} y^{2}}{x^{2}+y^{2}}+b_{1} \frac{x^{2}-y^{2}}{x^{2}+y^{2}}+b_{2}\right) \Psi=0 .
$$

where $b_{1}=\left(a_{2}-a_{1}\right) / 2, b_{2}=\left(a_{2}+a_{1}\right) / 2$. It defines an eigenvalue problem with spectral parameter $-b_{2}$. Applying the constraint equation $a_{4}=-$ $4 k_{3}\left(2 N+2 k_{1}+2 k_{2}+1\right)$ to the case where $b_{1}, k_{3}, a_{4}$ are fixed, we find polynomial solutions for

$$
b_{2}=-4 \frac{b_{1}^{2}}{\left(6-\frac{a_{4}}{2 k_{3}}+4 N\right)^{2}}-\frac{1}{64 k_{3}^{2}}\left(8 N k_{3}-a_{4}+4 k_{3}\right)\left(8 N k_{3}-a_{4}+12 k_{3}\right),
$$

$N=0,1,2 \ldots$.

To verify that system (27) is in fact a system on the 2 -sphere, real on the 2 -sheet hyperboloid, we note that $x, y$ are degenerate elliptic coordinates of type 2 . Indeed setting

$$
s_{1}+i s_{2}=-\frac{i}{x y}, s_{1}-i s_{2}=\frac{i}{4} \frac{\left(x^{2}+y^{2}\right)}{x y}, s_{3}=\frac{i}{2} \frac{\left(y^{2}-x^{2}\right)}{x y},
$$

we see that $s_{1}^{2}+s_{2}^{2}+s_{3}^{2}=1$ and (27) becomes

$$
\left(\Delta_{2}^{\prime}+4 b_{1} \frac{s_{3}}{\sqrt{s_{1}^{2}+s_{2}^{2}}}-\frac{a_{3}}{\left(s_{1}+i s_{2}\right)^{2}}+2 i \frac{a_{4}}{\left(s_{1}+i s_{2}\right) \sqrt{s_{1}^{2}+s_{2}^{2}}}-b_{2}\right) \Psi=0
$$

where $\Delta_{2}^{\prime}$ is the Laplace-Beltrami operator on the complex 2-sphere.

\section{Stäckel equivalent classical systems}

Some discussion of the relationship between Laplace-type and Helmholtztype classical superintegrable systems is in order. It is well known that trajectories of Stäckel equivalent systems are connected by swapping the role of parameters [22]. We use the classical system [211] to illustrate such a connection. The basic ideas apply to all superintegrable systems. The classical system [211], classical counterpart of (24), is described by the Hamiltonian

$$
\mathcal{H}_{211}=p_{x}^{2}+p_{y}^{2}+\frac{a_{1}}{x^{2}}+\frac{a_{2}}{y^{2}}-a_{3}\left(x^{2}+y^{2}\right)+a_{4} .
$$

where $p_{x}$ and $p_{y}$ are the canonical momenta associated with the variables $x$ and $y$, respectively. Consider the associated Helmholtz system $\mathcal{H}_{E 1}=E_{E 1}$ where

$$
\mathcal{H}_{E 1}=p_{x}^{2}+p_{y}^{2}+\frac{a_{1}}{x^{2}}+\frac{a_{2}}{y^{2}}-a_{3}\left(x^{2}+y^{2}\right),
$$


and $E_{E 1}=-a_{4}$. This system admits basis of 3 functionally independent 2 nd order constants of the motion $\left\{\mathcal{H}_{E 1}, \mathcal{L}_{1}\left(a_{1}, a_{2}, a_{3}\right), \mathcal{L}_{2}\left(a_{1}, a_{2}, a_{3}\right)\right\}$ where the dependence on the $a_{j}$ is linear. A classical trajectory can be characterized by the specific values $E_{E 1}, L_{1}^{(0)}, L_{2}^{(0)}$, assumed by the constants of the motion along the trajectories. The phase space $\left(x, y, p_{x}, p_{y}\right)$ for the trajectories is 4-dimensional and the equations

$$
\mathcal{H}_{E 1}=E_{E 1}, \mathcal{L}_{1}=L_{1}^{(0)}, \mathcal{L}_{2}=L_{2}^{(0)},
$$

determine 3 independent intersecting hypersurfaces in that space. The intersection is a curve on which the trajectory lies. The intersection can be computed explicitly and takes the form

$$
\begin{gathered}
f\left(x, y, a_{1}, a_{2}, a_{3}, E_{E 1}, L_{1}^{(0)}, L_{2}^{(0)}\right)=0 \\
p_{x}=g\left(x, y, a_{1}, a_{2}, a_{3}, E_{E 1}, L_{1}^{(0)}, L_{2}^{(0)}\right), p_{y}=h\left(x, y, a_{1}, a_{2}, a_{3}, E_{E 1}, L_{1}^{(0)}, L_{2}^{(0)}\right) .
\end{gathered}
$$

Now, the classical system [E16] Hamiltonian takes the form

$$
\mathcal{H}_{E 16}=\frac{1}{x^{2}+y^{2}}\left(p_{x}^{2}+p_{y}^{2}+\frac{a_{1}}{x^{2}}+\frac{a_{2}}{y^{2}}+a_{4}\right)=a_{3} .
$$

Under the Stäckel transformation from $E 1$ to $E 16$ the constants of the motion $\mathcal{L}_{1}\left(a_{3}\right), \mathcal{L}_{2}\left(a_{3}\right)$ for $E 1$ transform to $\mathcal{L}_{1}^{\prime}=\mathcal{L}_{1}\left(\mathcal{H}_{E 16}\right), \mathcal{L}_{2}^{\prime}=\mathcal{L}_{2}\left(\mathcal{H}_{E 16}\right)$, the corresponding constants of the motion for E16. Thus, for the choices $E_{E 16}=a_{3}, E_{E 1}=-a_{4}, \mathcal{L}_{1}^{\prime}=\mathcal{L}_{1}, \mathcal{L}_{2}^{\prime}=\mathcal{L}_{2}$ on the fixed trajectory. From this we conclude that equations (29) for the trajectories of $E 1$ are exactly the same as the equations for the trajectories of $E 16$.

There are differences, of course. The coordinates $(x, y)$ have entirely different meanings for the two systems. Moreover, changing the energy for one system corresponds to changing a parameter in the potential function for the other. As a simple example, the 2D Kepler problem and the 2D isotropic oscillator are Stäckel equivalent. However, the attractive oscillator corresponds to bounded (ellipsoidal) Kepler trajectories, the repulsive oscillator to unbounded (hyperbolic) trajectories, and the oscillator with zero force constant to parabolic Kepler trajectories.

Via a simple coordinate transformation the Stäckel transform relates the trajectories of (30) and (28) explicitly. However, when looking at the dynamics there is a subtlety on the time variable. In order to explain it let us consider the evolution of the vector position $\mathbf{r}$. For the system $E 1$ we have the equation

$$
\frac{d}{d t} \mathbf{r}=\left\{\mathcal{H}_{E 1}, \mathbf{r}\right\}=2\left(p_{x}, p_{y}\right),
$$

where $\{$,$\} stands for the Poisson bracket. Similarly for E16$

$$
\frac{d}{d \tau} \mathbf{r}=\left\{\mathbf{r}, \mathcal{H}_{E 16}\right\}=2 \frac{1}{x^{2}+y^{2}}\left(p_{x}, p_{y}\right) .
$$


Thus,

$$
d t=\frac{d \tau}{x^{2}(t)+y^{2}(t)} .
$$

The relation (31) can be generalized for any pair of Stäckel equivalent systems.

\section{Conclusions and discussion}

Bound state eigenfunctions of time independent Schrödinger operators typically have the form of the ground state eigenfunction times a polynomial. The study of these polynomial eigenspaces and their connection with special functions and orthogonal polynomials is an important area in mathematical physics. In this paper we have demonstrated that for the $442 \mathrm{D}$ quantum 2nd order superintegrable systems these polynomial spaces can be classified in an unified manner by transforming the quantum eigenvalue systems to 14 conformally superintegrable Laplace equations with potential. Multiple 2nd order Helmholtz superintegrable systems correspond to a single Laplace system and determination of formal ground states and polynomial eigenspaces of the Laplace system holds for all Helmholtz systems Stäckel equivalent to it. Also we showed that the possible R-separable coordinate systems for each Laplace equation automatically separate each associated Helmholtz system. We determined which of these 2D polynomial spaces is exactly solvable and which is quasi-exactly solvable and we determined the possible separable coordinate systems and associated special functions. Separation of variables in a 2D superintegrable system allowed us to construct one-dimensional QES system. We also showed how other 2D QES and PT symmetric systems on constant curvature spaces can be obtained as extensions of the solvable ones. For the associated classical mechanical 2D superintegrable systems we showed how the trajectories of Helmholtz systems that correspond to the same Laplace equation are related.

In other papers $[13,14,1,2]$ we have examined the effect of contracting superintegrable systems as it applies to the symmetry algebras of the systems. Thus we showed how the Askey scheme for orthogonal polynomials of hypergeometric type could be derived as sequences of contractions of the symmetry algebra of $S 9$, conformally Stäckel equivalent to $[1,1,1,1]$. Analogous procedures can be applied to the polynomial eigenspaces of the Helmholtz systems described here. They lead to limit relations for special functions of hypergeometric type, but also for nonhypergeometric Heun-type polynomial functions.

These ideas clearly apply to 3D 2nd order superintegrable systems on conformally flat spaces where the details are much more complicated. The systems with nondegenerate potentials have been classified, [9], but a classification of systems with degenerate 1 and 3-parameter potentials has never 
been undertaken. We will address these issues in other papers.

\section{Acknowledgment}

This work was partially supported by a grant from the Simons Foundation (\# 208754 to Willard Miller, Jr) and by CONACYT grant (\# 250881 to M.A. Escobar ).

\section{References}

[1] E. G. Kalnins, W. Miller Jr. and Eyal Subag, Laplace equations, conformal superintegrability and Bôcher contractions, Acta Polytechnica, (to appear ), (2016). arXiv:1510.09067 [math-ph]

[2] E. G. Kalnins, W. Miller Jr. and Eyal Subag, Bôcher contractions of conformally superintegrable Laplace equations, (submitted) arXiv:1512.09315 [math-ph], (2016); Bôcher contractions of conformally superintegrable Laplace equations: Detailed computations, arXiv:1601.02876 [math-ph], (2016).

[3] Bôcher, M., Ueber die Reihenentwickelungender Potentialtheorie, B. G. Teubner, Leipzig (1894).

[4] E. G. Kalnins, J. M. Kress, W. Miller, Jr. and S. Post, Laplacetype equations as conformal superintegrable systems, Adv. Appl. Math. (2011).

[5] Kalnins E.G., Kress J.M, and Miller W.Jr., Second order superintegrable systems in conformally flat spaces. II: The classical 2D Stäckel transform, J. Math. Phys., V.46, 053510, (2005).

[6] E. G. Kalnins, J. M. Kress and W. Miller Jr. Second order superintegrable systems in conformally flat spaces $\mathrm{V}: 2 \mathrm{D}$ and $3 \mathrm{D}$ quantum systems, J. Math. Phys., 47, 093501, (2006)

[7] W. Miller, Jr., S. Post and P. Winternitz, Classical and Quantum Superintegrability with Applications, J. Phys. A: Math. Theor., 46, (2013) 423001. (a 97 page topical review paper)

[8] E. G. Kalnins, J. M. Kress,W. Miller Jr. and G. S. Pogosyan, Completeness of superintegrability in two dimensional constant curvature spaces, J. Phys. A Math Gen. 34, 4705, (2001).

[9] Capel J.J. and Kress J.M., Invariant classification of second-order conformally flat superintegrable systems, J. Phys.A: Math. Theor, 47 (2014), 495202. 
[10] J. M. Kress, Equivalence of superintegrable systems in two dimensions, Physics of Atomic Nuclei, 70, No. 3, pp. 560566, (2007).

[11] E. G. Kalnins, W. Miller Jr. and S. Post, Wilson polynomials and the generic superintegrable system on the 2-sphere, J. Phys. A: Math. Theor. 40, 11525-11538 (2007), http://dx.doi.org/10.1088/1751$8113 / 40 / 38 / 005$

[12] Q. Li and W. Miller Jr., Wilson polynomials/functions and intertwining operators for the generic quantum superintegrable system on the 2-sphere, 2015 J. Phys.: Conf. Ser. 597012059 (http://iopscience.iop.org/1742-6596/597/1/012059)

[13] E. G. Kalnins, W. Miller Jr. and S. Post, Contractions of 2D 2nd order quantum superintegrable systems and the Askey scheme for hypergeometric orthogonal polynomials SIGMA, 9 057, 28 pages, (2013), http://dx.doi.org/10.3842/SIGMA.2013.057

[14] E. G. Kalnins and W. Miller Jr., Quadratic algebra contractions and 2nd order superintegrable systems, Anal. Appl. 12, 583-612, (2014), http://dx.doi.org/10.1142/S0219530514500377

[15] Tempesta P., Turbiner A.V. and P. Winternitz, Exact solvability of superintegrable systems, Journal of Math Physics, 42, (2001), 42484257

[16] A.V. Turbiner, Quasi-exactly-solvable problems and $\operatorname{sl}(2)$ algebra, Comm. Math. Phys., 118, (1988) 467.

[17] A.G.Ushveridze, Quasi-exactly solvable models in quantum mechanics Institute of Physics, Bristol, 1993.

[18] E.G. Kalnins, W. Miller, Jr. and and G.S. Pogosyan, Exact and quasiexact solvability of second order superintegrable quantum systems. I. Euclidean space preliminaries, J. Math. Phys., 47, 033502 (2006); Exact and quasi-exact solvability of second order superintegrable quantum systems. II. Connection with separation of variables, J. Math. Phys., 48, 023503 (2007)

[19] W. Rühl and A. V. Turbiner, Exact solvability of the Calogero and Sutherland models, Mod. Phys. Lett., A10, (1995), 2213-2222

[20] A. Mostafazadeh, Pseudo-Hermiticity versus PT symmetry: the necessary condition for the reality of the spectrum of a non-Hermitian Hamiltonian, J. Math. Phys., 43, (2002), 205-214, math-ph/0107001.

[21] J. Fris, V. Mandrosov, Ya. A. Smorodinsky, M. Uhlir and P. Winternitz, On higher symmetries in quantum mechanics, Phys.Lett., 16, (1965), 354-356 
[22] W. Miller Jr., E. G. Kalnins and S. Post, Coupling constant metamorphosis and Nth order symmetries in classical and quantum mechanics, J. Phys. A: Math. Theor., 43 (2010) 035202. (20 pages) 